\title{
Antibiotics did not prevent adverse outcomes after preterm, prelabour rupture of fetal membranes
}

Kenyon SL, Taylor DJ, Tarnow-Mordi W,for the ORACLE Collaborative Group. Broad-spectrum antibiotics for preterm, prelabour rupture of fetal membranes: the ORACLE I randomised trial. Lancet 2001 Mar 31;357:979-88.

\section{QUESTION: In pregnant women with preterm, prelabour rupture of fetal membranes (pPROM), are antibiotics better than placebo for preventing neonatal death, chronic lung disease, or major cerebral abnormality before discharge?}

Design

Randomised \{allocation concealed*\}†, blinded (patients and clinicians foutcome assessors and statisticians $\} \uparrow)$,* placebo controlled trial with a median follow up of 4 days.

Medical Research

Council. Co-amoxiclav

(Augmentin) and

co-amoxiclav placebo

were supplied by

SmithKline Beecham;

erythromycin (Erymax)

and erythromycin

placebo were supplied

by Parke Davis.

For correspondence:

Ms S Kenyon,

Department of

Obstetrics and

Gynaecology, Robert

Kilpatrick Building,

Leicester Royal

Infirmary, $P O$ Box 65 ,

Leicester LE2 7LX

UK. Fax $+44(0) 116$

2523154 .

\section{Setting}

161 centres in 15 countries.

\section{Patients}

4826 pregnant women who had fetuses that were $<37$ weeks of gestation, had PPROM, and had an uncertain need for antibiotics. Women who would have immediate or unstoppable delivery or who had fetuses not premature enough to warrant concern, had medical or drug contraindications, were prescribed antibiotics, or who were to be prescribed antibiotics for infection were excluded. 4809 women (mean age 28 y) were studied after exclusion of 15 women for protocol violations and 2 women for loss to follow up.
Erythromycin (Ery), co-amoxiclav (Co-amx), and combined treatments v placebo (Pl) for the composite outcome measure of death, chronic lung disease, or major cerebral abnormality in neonates of women who had pPROM*

\begin{tabular}{llcl} 
Comparison & Event rates & RRR $(95 \%$ CI) & NNT \\
Ery $v$ PI & $12.7 \% v 15.2 \%$ & $16.4 \%(-2.0$ to 31.5$)$ & Not significant \\
\hline Co-amx $v$ PI & $13.5 \% v 15.2 \%$ & $10.9 \%(-8.2$ to 26.7$)$ & Not significant \\
\hline Combined $v$ PI & $14.0 \% v 15.2 \%$ & $7.5 \%(-12.2$ to 23.7$)$ & Not significant \\
\hline
\end{tabular}

${ }^{*} \mathrm{pPROM}=$ preterm, prelabour rupture of fetal membranes. Other abbreviations defined in glossary; RRR, NNT, and $\mathrm{Cl}$ calculated from data in article.

\section{Intervention}

1197 women were allocated to erythromycin, $250 \mathrm{mg}$; 1212 were allocated to co-amoxiclav, $325 \mathrm{mg}$ (composed of amoxicillin, $250 \mathrm{mg}$, plus clavulanic acid, $125 \mathrm{mg}$ ); 1192 were allocated to combined co-amoxiclav, $325 \mathrm{mg}$, and erythromycin, $250 \mathrm{mg}$; and 1225 were allocated to placebo. All treatments were taken orally 4 times daily for 10 days or until delivery.

\section{Main outcome measure}

Composite measure of neonatal death, chronic lung disease (received daily supplementary oxygen at 36 weeks after conception), or major cerebral abnormality on ultrasonography before discharge.

\section{Main results}

Analysis was by intention to treat. Erythromycin, co-amoxiclav, and combined treatments were not better than placebo for preventing occurrence of the composite measure before discharge (table).

\section{Conclusion}

In pregnant women with preterm, prelabour rupture of fetal membranes, erythromycin, co-amoxiclav, or combined treatments were not better than placebo for preventing the composite outcome measure of neonatal death, chronic lung disease, or major cerebral abnormality before discharge.

*See glossary.

†Information provided by author.

\section{COMMENTARY}

At the beginning of the 21 st century, preterm delivery remains the major perinatal challenge. Even mild and moderate preterm birth infants are at high relative risk for death. ${ }^{1}$ Widespread uncertainty exists about the benefits and risks of antibiotic use for women in spontaneous labour with intact membranes and for those with pPROM. The findings of these studies by Kenyon et al (ORACLE I and II) are therefore highly relevant to practitioners and those planning future research.

The authors have enrolled unprecedented numbers of women in preterm labour in their placebo controlled trial $(>4$ times the number of women included in all previous randomised controlled trials in this area). Details are not given, however, of eligible patients who were not offered or who declined entry to the trial, and information is not available about the proportions of women in participating hospitals who were considered "ineligible" because antibiotics had already been prescribed or were considered necessary. If large numbers of women were already receiving antibiotics, this would limit the generalisability of the results, with those entered in ORACLE possibly representing a group who were "less at risk." This possibility may explain the finding that two thirds of the mothers in ORACLE II delivered at term as well as the finding of low overall mortality (about 2.5\%). In previous studies on antibiotics in preterm labour cited in the Cochrane review, the overall mortality was $15 \% .{ }^{2}$ Use of a predictive test, such as fetal fibronectin, may have allowed identification of a higher risk group. However, in ORACLE I, in which the entry criterion of "ruptured membranes" was more clearly defined, the mortality rate was about $6.3 \%$, which compares with the meta-analysed rate of $7.6 \%{ }^{3}$ 


\title{
Antibiotics did not prevent adverse outcomes in women in preterm labour with intact fetal membranes
}

\author{
Kenyon SL, Taylor DJ, Tarnow-Mordi W, for the ORACLE Collaborative Group. Broad-spectrum antibiotics for spontaneous \\ preterm labour: the ORACLE II randomised trial. Lancet 2001 Mar 31;357:989-94.

\section{QUESTION: For pregnant women in preterm labour with intact fetal membranes, are antibiotics better than placebo for preventing neonatal death, chronic lung disease, or major cerebral abnormality before discharge?}

\section{Design}

Randomised \{allocation concealed*\}†, blinded (patients and clinicians foutcome assessors and statisticians $\} \uparrow$ ), placebo controlled trial with a median follow up of 3 days.

\section{Setting}

161 centres in 15 countries.

\section{Patients}

6295 pregnant women who had fetuses that were $<37$ weeks of gestation, who were in suspected or definite preterm labour with intact fetal membranes, and who had an uncertain need for antibiotics. Women who would have immediate or unstoppable delivery or who had fetuses not premature enough to warrant concern, had medical or drug contraindications, were prescribed antibiotics, or who were to be prescribed antibiotics for infection were excluded. 6241 women (mean age 26 y) were studied after exclusion of 14 women for protocol violations and 40 women for loss to follow up.

\section{Intervention}

1611 women were allocated to erythromycin, $250 \mathrm{mg}$; 1550 were allocated to co-amoxiclav, $325 \mathrm{mg}$ (composed of amoxicillin, $250 \mathrm{mg}$, plus clavulanic acid, $125 \mathrm{mg}$ ); 1565 were allocated to combined co-amoxiclav, $325 \mathrm{mg}$, and erythromycin, $250 \mathrm{mg}$; and 1569 were allocated to placebo. All treatments were taken orally 4 times daily for 10 days or until delivery.

\section{Main outcome measure}

Composite measure of neonatal death, chronic lung disease (received daily supplementary oxygen at 36 weeks after conception), or major cerebral abnormality on ultrasonography before discharge.

\section{Main results}

Analysis was by intention to treat. Erythromycin, co-amoxiclav, and combined treatments were not better than placebo for preventing occurrence of the composite measure before discharge (table).

\section{Conclusion}

For pregnant women in preterm labour with intact fetal membranes, erythromycin, co-amoxiclav, or combined treatments were not better than placebo for preventing the composite outcome measure of neonatal death, chronic lung disease, or major cerebral abnormality before discharge.

*See glossary.

$\dagger$ Information provided by author.

Sources of funding: $U K$ Medical Research Council. Co-amoxiclav (Augmentin) and co-amoxiclav placebo were supplied by SmithKline Beecham; erythromycin (Erymax) and erythromycin placebo were supplied by Parke Davis.

For correspondence: Ms S Kenyon,

Department of Obstetrics and Gynaecology, Robert Kilpatrick Building, Leicester Royal Infirmary, P.O. Box 65 Leicester LE2 7LX,

UK. Fax +44 (0) 116 2523154.

Co-amoxiclav (Co-amx), erythromycin (Ery), and combined treatments v placebo (Pl) for the composite outcome measure of death, chronic lung disease, or major cerebral abnormality in neonates of women who were in preterm labour*

\begin{tabular}{llll} 
Comparison & Event rates & RRR $(95 \% \mathrm{Cl})$ & NNT \\
Co-amx $v$ PI & $4.95 \% v 5.01 \%$ & $1.2 \%(-34.3$ to 27.3$)$ & Not significant \\
& & RRI $(\mathrm{Cl})$ & NNH \\
\hline Ery $v$ PI & $5.63 \% v 5.01 \%$ & $12.2 \%(-16.4$ to 50.6$)$ & Not significant \\
\hline Combined $v$ PI & $5.87 \% v 5.01 \%$ & $17.0 \%(-12.7$ to 56.9$)$ & Not significant \\
\hline
\end{tabular}

*Abbreviations defined in glossary; RRR, RRI, NNT, NNH, and Cl calculated from data in article.

\section{COMMENTARY—continued from previous page}

Groups did not differ for the main outcome in either ORACLE I or II. Secondary analyses in women with pPROM suggested several benefits. Although these findings may indeed be the result of chance, ${ }^{4}$ they mirror trends seen in the meta-analysis. ${ }^{3}$ Given the growing body of evidence that intrauterine infection is linked to long term adverse outcome and the absence of findings to show harm associated with erythromycin treatment, it is highly likely that prophylactic treatment in this situation will become standard. Similarly, clinicians would be wise to avoid the use of augmentin (co-amoxiclav) in these situations.

The authors, in discussing the negative findings of ORACLE II, suggest future research in relation to screening for bacterial vaginosis and subsequent antibiotic treatment. It is worth noting that another trial with negative results has recently been published. ${ }^{5}$

Richard Johanson, MD, MA North Staffordshire Hospital Stoke-on-Trent, UK

1 Kramer MS, Demissie K, Yang H, et al. The contribution of mild and moderate preterm birth to infant mortality. Fetal and infant health study group of the Canadian perinatal surveillance system. JAMA 2000;284:843-9.

2 Kenyon S, Boulvain M. Antibiotics for preterm premature rupture of membranes. Cochrane Database Syst Rev 2001;(2):CD001058.

3 King J, Flenady V. Antibiotics for preterm labour with intact membranes. Cochrane Database Syst Rev 2001;(2):CD000246.

4 Hannah M. Antibiotics for preterm prelabour rupture of membranes and preterm labour? Lancet 2001;357:973-4.

5 Carey JC, Klebanoff MA, Hauth JC, et al. Metronidazole to prevent preterm delivery in pregnant women with asymptomatic bacterial vaginosis. National institute of child health and human development network of maternal-fetal medicine units. N Engl J Med 2000;342:534-40. 